\title{
Effect of Post Harvest Treatments on the Shelf life and Quality of Guava [Psidium guajava (L.)] cv. Allahabad Safeda
}

\author{
Afreen Tabasum ${ }^{1 *}$, Ch. Raja Goud ${ }^{2}$, Veena Joshi ${ }^{3}$, D. Anitha Kumari ${ }^{4}$ and A. Bhagwan ${ }^{4}$ \\ ${ }^{1}$ Department of Entomology, College of Horticulture, Sri Konda Laxman Telangana State \\ Horticulture University, Rajendranagar, Hyderabad-500030, Telangana, India \\ ${ }^{2}$ Department of Fruit Science, SKLTSHU, Rajendranagar, Hyderabad-500030, \\ Telangana, India \\ ${ }^{3}$ Vegetable Research Station, Rajendranagar, Hyderabad-500030, Telangana, India \\ ${ }^{4}$ Fruit Research Station, Sangareddy, Hyderabad, India \\ *Corresponding author
}

\section{A B S T R A C T}

\section{Keywords}

Guava, Shelf life, quality, $\mathrm{GA}_{3}$, Calcium chloride, Salicylic acid, Potassium permanganate

Article Info

Accepted: 17 December 2018 Available Online: 10 January 2019
An experiment was conducted to determine the effect of different post harvest treatments on the shelf life and quality of guava. Different chemicals such as Gibberellic acid, Calcium chloride, Salicylic acid and Potassium permanganate were used, individually and in combinations. The different concentrations of $\mathrm{GA}_{3}$ at 25 and $50 \mathrm{ppm}$ and combination with $\mathrm{KMnO}_{4}\left(5 \mathrm{~g} / \mathrm{kg}\right.$ sachet), $\mathrm{CaCl}_{2}$ at 1 and $2 \%$ and in combination with $\mathrm{KMnO}_{4}(5 \mathrm{~g} / \mathrm{kg}$ sachet) and Salicylic acid at $70 \& 140 \mathrm{ppm}$ and in combination with $\mathrm{KMnO}_{4}(5 \mathrm{~g} / \mathrm{kg}$ sachet $)$ was used. Guava fruits of cv. Allahabad Safeda were treated with chemicals and stored at ambient temperatures. Fruits which were treated with $140 \mathrm{ppm}$ salicylic acid i.e., $\mathrm{T}_{6}$ were significant among all the treatments and recorded lowest PLW (1.79\%), minimum fruit rotting (3.69\%), highest shelf life ( 7 days) over control (4 days). The $2^{\text {nd }}$ best treatment which was found to be significant was, fruits treated with salicylic acid at $70 \mathrm{ppm}$ i.e., $\mathrm{T}_{5}$. Biochemical parameters such as total sugars (6.75\%), reducing sugars (3.83) and TSS $\left(11.68^{\circ}\right.$ brix) were found to be significant and highest in the fruits treated with salicylic acid at $140 \mathrm{ppm}$. It was concluded that fruits treated with salicylic acid were found be effective in increasing the shelf life and quality of guava fruits.

\section{Introduction}

Guava is the $4^{\text {th }}$ most important fruit after Mango, Banana and Citrus and it is popularly known as the "Apple of the tropics". In India, the most important guava growing states are Uttar Pradesh, Bihar, Madhya Pradesh, Maharashtra and Gujarat etc. Uttar Pradesh is one of the most important states of India where, half of the total area is under guava production and district Allahabad has the reputation of growing the best guava in the country as well as in the world.

Salicylic acid is a plant hormone which inhibits ethylene biosynthesis and delays the 
senescence (Ozeker, 2005). It has been shown to inhibit the conversion of ACC into ethylene (Leslie and Romani, 1988) by suppressing ACC oxidase activity (Fan et al., 1996). It is also involved in local and systemic resistance to pathogens (Yalpani et al., 1994; Kang et al., 2003). Exogenous application of SA has been reported to delay the ripening of Peach (Han et al., 2003), Banana (Srivastava and Dwivedi, 2000).

Gibberellins $\left(\mathrm{GA}_{3}\right)$ are a group of growth substances, known to retard ripening and senescence of fruits. The effects of $\mathrm{GA}_{3}$ seem to be mainly on colour development, although other aspects of ripening processes are also affected. $\mathrm{GA}_{3}$ delays chlorophyll degradation and fruit softening (Vendrell, 1970) and decreases sugar accumulation, TSS and sugar/acid ratio in Banana (Ahmed and Tingwa, 1995) and Mango (Murthy and Rao, 1982).

Pre and post harvest application of calcium may delay senescence in fruits with no detrimental effect on consumer acceptance (Lester and Grusak, 2004). Exogenously applied Calcium stabilizes the plant cell wall and protects it from cell wall degrading enzymes (White and Broadley, 2003). Studies have shown that the rate of senescence often depends on the calcium status of the tissue and by increasing calcium levels, various parameters of senescence such as respiration, protein, chlorophyll content and membrane fluidity are altered (Poovaiah, 1984). It is also involved in reducing the rate of senescence and fruit ripening (Ferguson, 1984).

The inclusion of potassium permanganate, which is an ethylene absorbent, aims an extension of storage period (Salunkhe and Desai, 1984). It is quite effective in reducing ethylene levels by oxidizing it to carbon dioxide and water. It is a chemical which has been used to remove ethylene from storage atmosphere. The use of $\mathrm{KMnO}_{4}$ in conjunction with modified atmosphere in polyethylene films delayed fruit ripening, maintained quality and extended shelf life in Mango and Banana. Several studies have shown that $\mathrm{KMnO}_{4}$ applications delay fruit softening and increase post-harvest life (Illeperuma and Jayasuriya, 2002).

\section{Materials and Methods}

A lab experiment to investigate the effect of post harvest treatments on the shelf life and quality of guava (Psidium guajava L.) was carried out in Completely Randomized Design with three replications at Laboratory of fruit science, Department of Fruit Science, Sri Konda Laxman Telangana State Horticultural University, Rajendranagar, Hyderabad, during 2017-18. The experimental material comprised of fruits of cultivar Allahabad Safeda obtained from Fruit Research Station, Sangareddy, Hyderabad.

\section{Treatments}

$\mathrm{T}_{1}$ - Gibberellic acid @25 ppm

$\mathrm{T}_{2}$ - Gibberellic acid @ 50 ppm

$\mathrm{T}_{3}$ - Calcium chloride @1\%

$\mathrm{T}_{4}$ - Calcium chloride @ 2\%

$\mathrm{T}_{5}$ - Salicylic acid @70 ppm

$\mathrm{T}_{6}$ - Salicylic acid@140 ppm

$\mathrm{T}_{7}-$ Gibberellic acid $(25 \mathrm{ppm})+\mathrm{KMnO}_{4}$ ( $5 \mathrm{~g} / \mathrm{kg}$ sachet)

$\mathrm{T}_{8}-$ Gibberellic acid $(50 \mathrm{ppm})+\mathrm{KMnO}_{4}$ (5g/kg sachet)

$\mathrm{T}_{9}$ - Calcium chloride $(1 \%)+\mathrm{KMnO}_{4}(5 \mathrm{~g} / \mathrm{kg}$ sachet)

$\mathrm{T}_{10}$-Calcium chloride $(2 \%)+\mathrm{KMnO}_{4}(5 \mathrm{~g} / \mathrm{kg}$ sachet)

$\mathrm{T}_{11}$-Salicylic acid $(70 \mathrm{ppm})+\mathrm{KMnO}_{4}(5 \mathrm{~g} / \mathrm{kg}$ sachet)

$\mathrm{T}_{12}$ - Salicylic acid (140 ppm) $+\mathrm{KMnO}_{4}$ (5g/kg sachet)

$\mathrm{T}_{13}$ - Control 
Observations were recorded at 0 (initial), 2, 4 and 6 days interval. Observations on physicochemical characters and sensory evaluation of guava fruits with different treatments were recorded as per the standard methods given for different characters.

\section{Physiological loss in weight}

For determination of Physiological Loss in Weight (PLW), three fruits from each replication were marked and labeled. The marked and labeled fruits in each treatment were weighed prior to storage. Their weight was determined on 0 (initial), $2^{\text {nd }}, 4^{\text {th }}$ and $6^{\text {th }}$ day of storage. PLW was expressed on percent basis (on the basis of original weight of fruit).

\section{Rotting}

It was calculated by counting the number of decayed fruits from carton boxes at different intervals. Decayed fruits were weighed on the day of each observation. The percent rotting was estimated using the following formula:

\section{Shelf life}

After treating the guava fruits as per the treatments, they are whipped by muslin cloth and wrapped in double layer of newspaper and stored in carton boxes at room conditions up to 6 days. On the basis of fruit decay (\%), shelf life is considered.

\section{Sugars}

The sugars were estimated as per the method advocated by Ranganna (1979). 5g of pulp was macerated and transferred to $250 \mathrm{ml}$ volumetric flask, with $100 \mathrm{ml}$ of distilled water. $2 \mathrm{ml}$ saturated lead acetate was added to precipitate the tannin present in the sample. In the next step $25 \mathrm{ml}$ of saturated disodium hydrogen phosphate $\mathrm{Na}_{2} \mathrm{HPO}_{4}$ was added to precipitate excess amount of lead acetate. It was then shaked well and then filtered in 250 $\mathrm{ml}$ volumetric flask to make the volume 250 $\mathrm{ml}$. The extract (Aliquot) was then used for the estimation of sugars, as follows.

\section{Total sugars}

Total sugars were also estimated by the same method. After acid hydrolysis of $50 \mathrm{ml}$ aliquot with $5 \mathrm{ml}$ concentrated $\mathrm{HCl}$, it is mixed well and kept for $24 \mathrm{~h}$. It is then followed by neutralization with Sodium hydroxide using phenolphthalein indicator. Finally it is titrated against Fehling's solution using methylene blue indicator. The total sugars percentage was calculated using standard formula.

Total sugars $(\%)=$

Glucose equivalent $(0.05) \times$ Vol. made up $\times 100$

Titrate value $\times$ Weight of the sample

\section{Reducing sugars}

The prepared aliquot was then titrated against boiling standard Fehling's mixture $(5 \mathrm{ml}$ each Fehling's A and B solution) using methylene blue as an indicator until the sample has changed its colour to brick red precipitate. Reducing sugars percentage was calculated using standard formula.

Reducing sugars $(\%)=$

Glucose equivalent $(0.05) \times$ Vol. made up $\times 100$

Titrate value $\times$ Weight of the sample

\section{Total soluble solids}

Total soluble solids of the pulp was recorded using a hand refractometer in the range of $0-$ 32 percent. The juice was extracted from the fruit of guava and filtered through a cheese cloth and then the sample was taken on the 
prism of the hand refractometer. Three reading were taken for each replication and the average was considered. The results were expressed in terms of degree brix.

\section{Results and Discussion}

The Physiological loss in weight was significantly affected by various post harvest treatments in guava cv. Allahabad Safeda (Table 1). Under ambient conditions of storage, minimum average physiological loss in weight $(1.79 \%)$ was observed in the fruits which were treated with salicylic acid at 140 ppm $\left(T_{6}\right)$ which was on par with $T_{5}$ i.e., the fruits treated with salicylic acid at $70 \mathrm{ppm}$ (1.88\%). The maximum mean PLW $(2.48 \%)$ was recorded in $\mathrm{T}_{13}$ i.e., control. It is observed that SA treated fruits have positive effects in maintaining membrane integrity. Abbasi et al., (2010) observed less chilling injury and less weight loss than other treatments in fruits of peach treated with $1 \mathrm{mM}$ SA. Brar et al., (2014) found that $200 \mathrm{ppm}$ SA significantly reduced the PLW loss in peach fruit under cold storage condition.

Table.1 Effect of different post harvest treatments on physiological loss in weight (\%) in Guava cv. Allahabad Safeda

\begin{tabular}{|c|c|c|c|c|c|}
\hline \multirow[t]{3}{*}{ S.No. } & \multirow[t]{3}{*}{ Treatments } & \multicolumn{4}{|c|}{ PLW (\%) } \\
\hline & & \multicolumn{4}{|c|}{ Days after storage } \\
\hline & & 2 & 4 & 6 & Mean \\
\hline 1. & $\mathrm{~T}_{1}\left(\mathrm{GA}_{3} @ 25 \mathrm{ppm}\right)$ & 0.95 & 2.70 & 3.47 & 2.37 \\
\hline 2. & $\mathrm{~T}_{2}\left(\mathrm{GA}_{3} @ 50 \mathrm{ppm}\right)$ & 0.94 & 2.64 & 3.38 & 2.32 \\
\hline 3. & $\mathrm{~T}_{3}\left(\mathrm{CaCl}_{2} @ 1 \%\right)$ & 0.85 & 2.47 & 2.92 & 2.08 \\
\hline 4. & $\mathrm{~T}_{4} \quad\left(\mathrm{CaCl}_{2} @ 2 \%\right)$ & 0.87 & 2.42 & 2.87 & 2.05 \\
\hline 5. & $\mathrm{~T}_{5}$ (SA@ @ $\left.70 \mathrm{ppm}\right)$ & 0.80 & 2.30 & 2.54 & 1.88 \\
\hline 6. & $\mathrm{~T}_{6}(\mathrm{SA} @ 140 \mathrm{ppm})$ & 0.72 & 2.24 & 2.41 & 1.79 \\
\hline 7. & $\begin{array}{ll}\mathrm{T}_{7} & \mathrm{GA}_{3}(25 \mathrm{ppm})+ \\
& \mathrm{KMnO}_{4}(5 \mathrm{~g})\end{array}$ & 0.93 & 2.70 & 3.32 & 2.32 \\
\hline 8. & $\begin{array}{ll}\mathrm{T}_{8} & \mathrm{GA}_{3}(50 \mathrm{ppm})+ \\
\mathrm{KMnO}_{4}(5 \mathrm{~g})\end{array}$ & 0.90 & 2.60 & 3.24 & 2.25 \\
\hline 9. & $\begin{array}{ll}\mathrm{T}_{9} & \mathrm{CaCl}_{2}(1 \%)+ \\
& \mathrm{KMnO}_{4}(5 \mathrm{~g})\end{array}$ & 0.89 & 2.55 & 3.14 & 2.19 \\
\hline 10. & $\begin{array}{c}\mathrm{T}_{10} \mathrm{CaCl}_{2}(2 \%)+ \\
\mathrm{KMnO}_{4}(5 \mathrm{~g})\end{array}$ & 0.87 & 2.50 & 3.04 & 2.14 \\
\hline 11. & $\begin{array}{rl}\mathrm{T}_{11} & \mathrm{SA}(70 \mathrm{ppm})+ \\
& \mathrm{KMnO}_{4}(5 \mathrm{~g})\end{array}$ & 0.84 & 2.32 & 2.64 & 1.93 \\
\hline 12. & $\begin{array}{l}\mathrm{T}_{12} \\
\mathrm{SA}(140 \mathrm{ppm})+ \\
\mathrm{KMnO}_{4}(5 \mathrm{~g})\end{array}$ & 0.86 & 2.38 & 2.69 & 1.98 \\
\hline 13. & $\mathrm{~T}_{13} \quad$ (Control) & 0.97 & 2.70 & 3.76 & 2.48 \\
\hline \multirow{2}{*}{\multicolumn{2}{|c|}{ Mean }} & 0.88 & 2.50 & 3.03 & 2.14 \\
\hline & & 2 & 4 & \multicolumn{2}{|l|}{ J.0J } \\
\hline & $\mathrm{SE}(\mathrm{m}) \pm$ & 0.01 & 0.02 & \multicolumn{2}{|c|}{0.04} \\
\hline & CD at $5 \%$ & 0.04 & 0.08 & \multicolumn{2}{|c|}{0.13} \\
\hline
\end{tabular}


Table.2 Effect of different post harvest treatments on fruit rotting (\%) in Guava cv. Allahabad Safeda

\begin{tabular}{|c|c|c|c|c|c|}
\hline \multirow[t]{3}{*}{ S.No. } & \multirow[t]{3}{*}{ Treatments } & \multicolumn{4}{|c|}{ Fruit rotting (\%) } \\
\hline & & \multicolumn{4}{|c|}{ Days after storage } \\
\hline & & 2 & 4 & 6 & Mean \\
\hline 1. & $\mathrm{~T}_{1}\left(\mathrm{GA}_{3} @ 25 \mathrm{ppm}\right)$ & 0.00 & 7.89 & 11.26 & 6.38 \\
\hline 2. & $\mathrm{~T}_{2}\left(\mathrm{GA}_{3} @ 50 \mathrm{ppm}\right)$ & 0.00 & 7.47 & 10.73 & 6.07 \\
\hline 3. & $\mathrm{~T}_{3}\left(\mathrm{CaCl}_{2} @ 1 \%\right)$ & 0.00 & 5.54 & 8.62 & 4.72 \\
\hline 4. & $\mathrm{~T}_{4}\left(\mathrm{CaCl}_{2} @ 2 \%\right)$ & 0.00 & 5.29 & 8.58 & 4.62 \\
\hline 5. & $\mathrm{~T}_{5}(\mathrm{SA} @ 70 \mathrm{ppm})$ & 0.00 & 4.72 & 7.23 & 3.98 \\
\hline 6. & $\mathrm{~T}_{6} \quad(\mathrm{SA} @ 140 \mathrm{ppm})$ & 0.00 & 4.06 & 7.00 & 3.69 \\
\hline 7. & $\begin{array}{l}\mathrm{T}_{7} \quad \mathrm{GA}_{3}(25 \mathrm{ppm})+\mathrm{KMnO}_{4} \\
(5 \mathrm{~g})\end{array}$ & 0.00 & 7.29 & 10.99 & 6.09 \\
\hline 8. & $\begin{array}{l}\mathrm{T}_{8} \mathrm{GA}_{3}(50 \mathrm{ppm})+\mathrm{KMnO}_{4} \\
(5 \mathrm{~g})\end{array}$ & 0.00 & 7.01 & 10.56 & 5.86 \\
\hline 9. & $\begin{array}{l}\mathrm{T}_{9} \quad \mathrm{CaCl}_{2}(1 \%)+\mathrm{KMnO}_{4} \\
(5 \mathrm{~g})\end{array}$ & 0.00 & 5.93 & 9.01 & 4.98 \\
\hline 10. & $\begin{array}{l}\mathrm{T}_{10} \mathrm{CaCl}_{2}(2 \%)+\mathrm{KMnO}_{4} \\
(5 \mathrm{~g})\end{array}$ & 0.00 & 5.84 & 8.94 & 4.93 \\
\hline 11. & $\begin{array}{l}\mathrm{T}_{11} \mathrm{SA}(70 \mathrm{ppm})+\mathrm{KMnO}_{4} \\
(5 \mathrm{~g})\end{array}$ & 0.00 & 4.82 & 8.07 & 4.30 \\
\hline 12. & $\begin{array}{l}\mathrm{T}_{12} \mathrm{SA}(140 \mathrm{ppm})+\mathrm{KMnO}_{4} \\
(5 \mathrm{~g})\end{array}$ & 0.00 & 4.88 & 8.32 & 4.40 \\
\hline 13. & $\mathrm{~T}_{13}$ (Control) & 0.00 & 9.64 & 14.62 & 8.09 \\
\hline \multirow{2}{*}{\multicolumn{2}{|c|}{ Mean }} & 0.00 & 6.18 & 9.53 & 5.24 \\
\hline & & 2 & 4 & \multicolumn{2}{|c|}{6} \\
\hline & $\mathrm{SE}(\mathrm{m}) \pm$ & 0.00 & 0.01 & \multicolumn{2}{|c|}{0.02} \\
\hline & CD at $5 \%$ & 0.00 & 0.04 & \multicolumn{2}{|c|}{0.07} \\
\hline
\end{tabular}


Table.3 Effect of different post harvest treatments on fruit shelf life (days) in Guava cv. Allahabad Safeda

\begin{tabular}{|c|c|c|}
\hline S.No. & Treatments & Shelf life (days) \\
\hline 1. & $\mathrm{~T}_{1}\left(\mathrm{GA}_{3} @ 25 \mathrm{ppm}\right)$ & 5.10 \\
\hline 2. & $\mathrm{~T}_{2}\left(\mathrm{GA}_{3} @ 50 \mathrm{ppm}\right)$ & 5.22 \\
\hline 3. & $\mathrm{~T}_{3}\left(\mathrm{CaCl}_{2} @ 1 \%\right)$ & 5.20 \\
\hline 4. & $\mathrm{~T}_{4}\left(\mathrm{CaCl}_{2} @ 2 \%\right)$ & 5.50 \\
\hline 5. & $\mathrm{~T}_{5}$ (SA@ @ 70 ppm $)$ & 6.50 \\
\hline 6. & $\mathrm{~T}_{6}(\mathrm{SA} @ 140$ ppm $)$ & 7.00 \\
\hline 7 & $\begin{array}{l}\mathrm{T}_{7} \quad \mathrm{GA}_{3}(25 \mathrm{ppm})+ \\
\mathrm{KMnO}_{4}(5 \mathrm{~g})\end{array}$ & 5.10 \\
\hline 8. & $\begin{array}{l}\mathrm{T}_{8} \mathrm{GA}_{3}(50 \mathrm{ppm})+ \\
\mathrm{KMnO}_{4}(5 \mathrm{~g})\end{array}$ & 5.20 \\
\hline 9. & $\begin{array}{l}\mathrm{T}_{9} \quad \mathrm{CaCl}_{2}(1 \%)+\mathrm{KMnO}_{4} \\
(5 \mathrm{~g})\end{array}$ & 5.50 \\
\hline 10. & $\begin{array}{l}\mathrm{T}_{10} \mathrm{CaCl}_{2}(2 \%)+\mathrm{KMnO}_{4} \\
(5 \mathrm{~g})\end{array}$ & 5.60 \\
\hline 11. & $\begin{array}{l}\mathrm{T}_{11} \mathrm{SA}(70 \mathrm{ppm})+\mathrm{KMnO}_{4} \\
(5 \mathrm{~g})\end{array}$ & 6.00 \\
\hline 12. & $\begin{array}{l}\mathrm{T}_{12} \mathrm{SA}(140 \mathrm{ppm})+ \\
\mathrm{KMnO}_{4}(5 \mathrm{~g})\end{array}$ & 6.00 \\
\hline 13. & $\mathrm{~T}_{13}$ (Control) & 4.00 \\
\hline & Mean & 5.53 \\
\hline & $\mathrm{SE}(\mathrm{m}) \pm$ & 0.160 \\
\hline & CD at $5 \%$ & 0.465 \\
\hline
\end{tabular}


Table.4 Effect of different post harvest treatments on total sugars (\%) in Guava cv. Allahabad Safeda

\begin{tabular}{|c|c|c|c|c|c|c|}
\hline \multirow{3}{*}{$\begin{array}{c}\text { S.N } \\
\text { o. }\end{array}$} & \multirow[t]{3}{*}{ Treatments } & \multicolumn{5}{|c|}{ Total sugars (\%) } \\
\hline & & \multicolumn{5}{|c|}{ Days after storage } \\
\hline & & $\mathbf{0}$ & 2 & 4 & 6 & Mean \\
\hline 1. & $\mathrm{~T}_{1}\left(\mathrm{GA}_{3} @ 25 \mathrm{ppm}\right)$ & 5.70 & 5.78 & 6.02 & 5.60 & 5.78 \\
\hline 2. & $\mathrm{~T}_{2}\left(\mathrm{GA}_{3} @ 50 \mathrm{ppm}\right)$ & 5.76 & 5.79 & 5.98 & 5.70 & 5.81 \\
\hline 3. & $\mathrm{~T}_{3}\left(\mathrm{CaCl}_{2} @ 1 \%\right)$ & 6.11 & 6.19 & 6.46 & 6.30 & 6.27 \\
\hline 4. & $\mathrm{~T}_{4}\left(\mathrm{CaCl}_{2} @ 2 \%\right)$ & 6.19 & 6.30 & 6.61 & 6.42 & 6.38 \\
\hline 5. & $\mathrm{~T}_{5} \quad(\mathrm{SA} @ 70$ ppm $)$ & 6.42 & 6.57 & 6.81 & 6.72 & 6.63 \\
\hline 6. & $\mathrm{~T}_{6}(\mathrm{SA} @ 140 \mathrm{ppm})$ & 6.53 & 6.73 & 6.96 & 6.77 & 6.75 \\
\hline 7. & $\begin{array}{l}\mathrm{T}_{7} \mathrm{GA}_{3}(25 \mathrm{ppm})+ \\
\mathrm{KMnO}_{4}(5 \mathrm{~g})\end{array}$ & 5.82 & 5.84 & 5.99 & 5.79 & 5.86 \\
\hline 8. & $\begin{array}{l}\mathrm{T}_{8} \quad \mathrm{GA}_{3}(50 \mathrm{ppm})+ \\
\mathrm{KMnO}_{4}(5 \mathrm{~g})\end{array}$ & 5.82 & 5.93 & 6.15 & 5.90 & 5.95 \\
\hline 9. & $\begin{array}{l}\mathrm{T}_{9} \mathrm{CaCl}_{2}(1 \%)+ \\
\mathrm{KMnO}_{4}(5 \mathrm{~g})\end{array}$ & 5.96 & 6.07 & 6.33 & 6.15 & 6.13 \\
\hline 10. & $\begin{array}{l}\mathrm{T}_{10} \mathrm{CaCl}_{2}(2 \%)+ \\
\mathrm{KMnO}_{4}(5 \mathrm{~g})\end{array}$ & 6.06 & 6.17 & 6.47 & 6.29 & 6.25 \\
\hline 11. & $\begin{array}{l}\mathrm{T}_{11} \mathrm{SA}(70 \mathrm{ppm})+ \\
\mathrm{KMnO}_{4}(5 \mathrm{~g})\end{array}$ & 6.39 & 6.50 & 6.80 & 6.61 & 6.58 \\
\hline 12. & $\begin{array}{l}\mathrm{T}_{12} \mathrm{SA}_{1}(140 \mathrm{ppm})+ \\
\mathrm{KMnO}_{4}(5 \mathrm{~g})\end{array}$ & 6.28 & 6.36 & 6.67 & 6.46 & 6.44 \\
\hline 13. & $\mathrm{~T}_{13}$ (Control) & 5.60 & 5.74 & 5.88 & 5.59 & 5.70 \\
\hline \multirow{2}{*}{\multicolumn{2}{|c|}{ Mean }} & 6.05 & 6.15 & 6.39 & 6.18 & 6.19 \\
\hline & & 0 & 2 & 4 & \multicolumn{2}{|c|}{6} \\
\hline & $\mathrm{SE}(\mathrm{m}) \pm$ & 0.02 & 0.02 & 0.05 & \multicolumn{2}{|c|}{0.02} \\
\hline & CD at $5 \%$ & 0.05 & 0.07 & 0.17 & \multicolumn{2}{|c|}{0.07} \\
\hline
\end{tabular}


Table.5 Effect of different post harvest treatments on reducing sugars (\%) in Guava cv. Allahabad Safeda

\begin{tabular}{|c|c|c|c|c|c|c|}
\hline \multirow[t]{3}{*}{ S.No. } & \multirow[t]{3}{*}{ Treatments } & \multicolumn{5}{|c|}{ Reducing sugars (\%) } \\
\hline & & \multicolumn{5}{|c|}{ Days after storage } \\
\hline & & $\mathbf{0}$ & 2 & 4 & 6 & Mean \\
\hline 1. & $\mathrm{~T}_{1}\left(\mathrm{GA}_{3} @ 25 \mathrm{ppm}\right)$ & 3.38 & 3.40 & 3.49 & 3.30 & 3.39 \\
\hline 2. & $\mathrm{~T}_{2}\left(\mathrm{GA}_{3} @ 50 \mathrm{ppm}\right)$ & 3.40 & 3.45 & 3.62 & 3.41 & 3.47 \\
\hline 3. & $\mathrm{~T}_{3}\left(\mathrm{CaCl}_{2} @ 1 \%\right)$ & 3.48 & 3.52 & 3.67 & 3.62 & 3.57 \\
\hline 4. & $\mathrm{~T}_{4}\left(\mathrm{CaCl}_{2} @ 2 \%\right)$ & 3.52 & 3.58 & 3.79 & 3.68 & 3.64 \\
\hline 5. & $\mathrm{~T}_{5}(\mathrm{SA} @ 70 \mathrm{ppm})$ & 3.62 & 3.68 & 3.84 & 3.83 & 3.74 \\
\hline 6. & $\mathrm{~T}_{6}(\mathrm{SA} @ 140 \mathrm{ppm})$ & 3.71 & 3.75 & 3.98 & 3.87 & 3.83 \\
\hline 7. & $\begin{array}{l}\mathrm{T}_{7} \mathrm{GA}_{3}(25 \mathrm{ppm})+ \\
\mathrm{KMnO}_{4}(5 \mathrm{~g})\end{array}$ & 3.35 & 3.39 & 3.51 & 3.42 & 3.42 \\
\hline 8. & $\begin{array}{l}\mathrm{T}_{8} \mathrm{GA}_{3}(50 \mathrm{ppm})+ \\
\mathrm{KMnO}_{4}(5 \mathrm{~g})\end{array}$ & 3.40 & 3.44 & 3.58 & 3.47 & 3.47 \\
\hline 9. & $\begin{array}{l}\mathrm{T}_{9} \mathrm{CaCl}_{2}(1 \%) \\
+\mathrm{KMnO}_{4}(5 \mathrm{~g})\end{array}$ & 3.44 & 3.50 & 3.64 & 3.59 & 3.54 \\
\hline 10. & $\begin{array}{l}\mathrm{T}_{10} \mathrm{CaCl}_{2}(2 \%)+ \\
\mathrm{KMnO}_{4}(5 \mathrm{~g})\end{array}$ & 3.49 & 3.54 & 3.72 & 3.66 & 3.60 \\
\hline 11. & $\begin{array}{l}\mathrm{T}_{11} \mathrm{SA}(70 \mathrm{ppm})+ \\
\mathrm{KMnO}_{4}(5 \mathrm{~g})\end{array}$ & 3.60 & 3.66 & 3.84 & 3.78 & 3.72 \\
\hline 12. & $\begin{array}{l}\mathrm{T}_{12} \mathrm{SA}(140 \mathrm{ppm})+ \\
\mathrm{KMnO}_{4}(5 \mathrm{~g})\end{array}$ & 3.56 & 3.60 & 3.78 & 3.70 & 3.66 \\
\hline 13. & $\mathrm{~T}_{13}$ (Control) & 3.32 & 3.38 & 3.41 & 3.29 & 3.35 \\
\hline \multirow{2}{*}{\multicolumn{2}{|c|}{ Mean }} & 3.48 & 3.53 & 3.68 & 3.59 & 3.57 \\
\hline & & 0 & 2 & 4 & \multicolumn{2}{|c|}{6} \\
\hline & $\mathrm{SE}(\mathbf{m}) \pm$ & 0.01 & 0.02 & 0.05 & \multicolumn{2}{|c|}{0.03} \\
\hline & CD at $5 \%$ & 0.029 & 0.06 & 0.16 & \multicolumn{2}{|c|}{0.08} \\
\hline
\end{tabular}


Table.6 Effect of different post harvest treatments on total soluble solids ( ${ }^{\circ}$ Brix) in Guava cv. Allahabad Safeda

\begin{tabular}{|c|c|c|c|c|c|c|}
\hline \multirow[t]{3}{*}{ S.No. } & \multirow[t]{3}{*}{ Treatments } & \multicolumn{5}{|c|}{ Total soluble solids $\left({ }^{\circ}\right.$ Brix) } \\
\hline & & \multicolumn{5}{|c|}{ Days after storage } \\
\hline & & $\mathbf{0}$ & 2 & 4 & 6 & Mean \\
\hline 1. & $\mathrm{~T}_{1}\left(\mathrm{GA}_{3} @ 25 \mathrm{ppm}\right)$ & 11.54 & 11.61 & 11.69 & 11.51 & 11.59 \\
\hline 2. & $\mathrm{~T}_{2}\left(\mathrm{GA}_{3} @ 50 \mathrm{ppm}\right)$ & 11.55 & 11.62 & 11.71 & 11.52 & 11.60 \\
\hline 3. & $\mathrm{~T}_{3}\left(\mathrm{CaCl}_{2} @ 1 \%\right)$ & 11.58 & 11.65 & 11.73 & 11.55 & 11.63 \\
\hline 4. & $\mathrm{~T}_{4}\left(\mathrm{CaCl}_{2} @ 2 \%\right)$ & 11.59 & 11.64 & 11.74 & 11.56 & 11.63 \\
\hline 5. & $\mathrm{~T}_{5}(\mathrm{SA} @ 70$ ppm $)$ & 11.62 & 11.68 & 11.77 & 11.58 & 11.66 \\
\hline 6. & $\mathrm{~T}_{6}(\mathrm{SA} @ 140 \mathrm{ppm})$ & 11.63 & 11.69 & 11.79 & 11.59 & 11.68 \\
\hline 7. & $\begin{array}{c}\mathrm{T}_{7} \mathrm{GA}_{3}(25 \mathrm{ppm})+ \\
\mathrm{KMnO}_{4}(5 \mathrm{~g})\end{array}$ & 11.55 & 11.62 & 11.70 & 11.53 & 11.60 \\
\hline 8. & $\begin{array}{c}\mathrm{T}_{8} \mathrm{GA}_{3}(50 \mathrm{ppm})+ \\
\mathrm{KMnO}_{4}(5 \mathrm{~g})\end{array}$ & 11.57 & 11.63 & 11.71 & 11.55 & 11.62 \\
\hline 9. & $\begin{array}{cc}\mathrm{T}_{9} & \mathrm{CaCl}_{2}(1 \%)+ \\
\mathrm{KMnO}_{4}(5 \mathrm{~g})\end{array}$ & 11.58 & 11.64 & 11.72 & 11.55 & 11.62 \\
\hline 10. & $\begin{array}{r}\mathrm{T}_{10} \mathrm{CaCl}_{2}(2 \%)+ \\
\mathrm{KMnO}_{4}(5 \mathrm{~g})\end{array}$ & 11.59 & 11.64 & 11.73 & 11.57 & 11.63 \\
\hline 11. & $\begin{array}{c}\mathrm{T}_{11} \mathrm{SA}(70 \mathrm{ppm})+ \\
\mathrm{KMnO}_{4}(5 \mathrm{~g})\end{array}$ & 11.61 & 11.67 & 11.76 & 11.58 & 11.66 \\
\hline 12. & $\begin{array}{c}\mathrm{T}_{12} \mathrm{SA}(140 \mathrm{ppm})+ \\
\mathrm{KMnO}_{4}(5 \mathrm{~g})\end{array}$ & 11.60 & 11.66 & 11.75 & 11.57 & 11.65 \\
\hline 13. & $\mathrm{~T}_{13}$ (Control) & 11.54 & 11.60 & 11.68 & 11.50 & 11.58 \\
\hline \multirow{2}{*}{\multicolumn{2}{|c|}{ Mean }} & 11.58 & 11.64 & 11.73 & 11.55 & 11.63 \\
\hline & & 0 & 2 & 4 & \multicolumn{2}{|c|}{6} \\
\hline & $\mathrm{SE}(\mathrm{m}) \pm$ & 0.006 & 0.009 & 0.011 & \multicolumn{2}{|c|}{0.004} \\
\hline & CD at $5 \%$ & 0.017 & 0.027 & 0.032 & \multicolumn{2}{|c|}{0.012} \\
\hline
\end{tabular}


Fatemi et al., (2013) observed that Salicylic acid application significantly decreased weight loss percentage and increased storage life of kiwi fruits. Similar results were reported by Abbasi et al., (2010), Brar et al., (2014) and Fatemi et al., (2013).

The rotting percent was significantly affected by various post harvest treatments in guava cv. Allahabad Safeda (Table 2). Under ambient conditions of storage, minimum rotting $(3.69 \%)$ was observed in the fruits treated with salicylic acid at $140 \mathrm{ppm}$ i.e. $\mathrm{T}_{6}$ which was on par with $\mathrm{T}_{5}$ i.e. fruits treated with salicylic acid at $70 \mathrm{ppm}(3.98 \%)$. The maximum rotting $(8.09 \%)$ was recorded in control. A rapid decay in control fruits at both room and low temperature storage conditions was reported (Ray et al., 2004). Fatemi et al., (2013) observed that SA at all concentrations inhibited grey mould growth in kiwi fruits. Litchi fruits kept at ambient storage conditions have got rotten after 4 days of storage (Marboh, 2009). Similar results were reported by Ray et al., 2004, Fatemi et al., 2013 and Marboh, 2009. The shelf life of fruits had significant difference on the post harvest treatments in guava fruits (Table 3). Highest shelf life (7 days) was recorded in fruits treated with salicylic acid at $140 \mathrm{ppm}$ i.e. $\mathrm{T}_{6}$ which was on par with $\mathrm{T}_{5}$ i.e. fruits treated with salicylic acid at $70 \mathrm{ppm}$ (6.5 days). Lowest shelf life (4 days) was recorded in control i.e. $\mathrm{T}_{13}$. The post harvest treatment with $5.0 \mathrm{mM}$ SA delayed the occurrence of IB in pineapple, extended its shelf life (Lu et al., (2010). Treatment of strawberry plants with SA at vegetative stage and fruit development stage followed by post harvest treatment of fruits with 1 and 2 mmol $\mathrm{L}^{-1}$ effectively controlled the total decay and increased shelf life (Babalar et al., 2007). Similar results were reported by $\mathrm{Lu}$ et al., (2010) and Babalar et al., (2007).

The total sugars had significant difference among various treatments in the fruits. The total sugars increased gradually from 0 to $4^{\text {th }}$ day of storage, by reaching its peak at $4^{\text {th }}$ day and then gradually declined from $6^{\text {th }}$ day onwards (Table 4). Highest total sugars $(6.75 \%)$ were recorded in $\mathrm{T}_{6}$ i.e. when the fruits were treated with salicylic acid at 140 ppm which was on par with $\mathrm{T}_{5}$ i.e. when the fruits were treated with salicylic acid at 70 ppm (6.63). Lowest total sugars were recorded in $\mathrm{T}_{13}$ i.e. in control. There was a sharp decline in total sugars in untreated fruits. Similar results were reported by $\mathrm{Lu}$ et al., (2011) in Pineapple and Sayyari et al., (2009) in Pomegranate.

Among all the treatments maximum reducing sugars $(3.83 \%)$ were recorded in $\mathrm{T}_{6}$ i.e. fruits treated with salicylic acid at $140 \mathrm{ppm}$ followed by $\mathrm{T}_{5}$ i.e. fruits treated with salicylic acid at $70 \mathrm{ppm}$. while minimum reducing sugars were recorded in $\mathrm{T}_{13}$ i.e. in control (Table 5). Reduction in reducing sugars level in untreated fruits was mainly due to higher rate of respiration which leads to unchecked progression of ripening and senescence. SA is well known phenol that can prevent ACO activity was suggested by Paliyath and Subramanian (2008). Salicylic acid treated fruits increased the reducing sugars in concentration manner in banana (Manoj and Upendra 2000). Similar results were reported by Manoj and Upendra (2000).

Highest Total soluble solids $\left(11.68{ }^{\circ} \mathrm{B}\right)$ was recorded in the fruits treated with salicylic acid at $140 \mathrm{ppm}$ i.e. $\mathrm{T}_{6}$ which was on par with $\mathrm{T}_{5}$ i.e. fruits treated with salicylic acid at 70 ppm $\left(11.66^{\circ} \mathrm{B}\right)$. Lowest TSS was recorded in control $\left(11.58{ }^{\circ} \mathrm{B}\right)$ (Table 6). Increase in the TSS of fruits may be due to reduction of the activities of various enzymes and by delaying senescence, disorganization of cellular structure and checking of microbial activities (Lougheed et al., 1979). The TSS and sugars increase during storage due to hydrolysis of starch into sugars as on complete hydrolysis of starch no further increase occurs and 
subsequently a decline in TSS is predictable as they along with other organic acids are primary substrate for respiration (Wills et al., 1980). Similar results were recorded by Fatemi et al., (2013) in kiwi fruits, when the fruits treated with SA at $5 \mathrm{mM}$ concentration had highest TSS. Hajilou et al., (2013) recorded highest TSS in $2.0 \mathrm{mM}$ and $3.0 \mathrm{mM}$ SA treatments in apricot.

It can be concluded that salicylic acid at 140 ppm was found to be the best among all the treatments in extending the shelf life and quality of guava cv. Allahabad Safeda.

\section{References}

Abbasi, N.A., Hafeez, S., Tareen, M.J., Hewett, E.W., Johnston, J.W and Gunson, F. 2010. Salicylic acid prolongs shelf life and improves quality of 'Maria Delicia' peach fruit. Acta Horticulturae, 880: 91-97.

Ahmed, O.K. and Tingwa, P.O. 1995. Effect of maleic hydrazide and waxing on ripening and quality of tomato fruits. University of Khartoum Journal of Agricultural Sciences, 3(1): 59-72.

Babalar, M., Asghari, M., Talaei, A. and Khosroshahi, A. 2007. Effect of pre and post-harvest salicylic acid treatment on ethylene production, fungal decay and overall quality of Selva strawberry fruit. Food Chemistry, 105: 449-53.

Brar, J.S., Gupta, N. and Gill, M.S. 2014. Effect of pre and post-harvest treatments of salicylic acid on quality characteristics of peach (Prunus persica L.) fruits during storage. Progressive Horticulture, 46: 217-21.

Fan, X., Matches, J.P. and Fellowman J.K. 1996. Inhibition of apple fruit 1aminocyclopropane-1-carboxylic acid oxides activity and respiration by acetylsalicylic acid. Journal of plant physiology, 149: 469-471.
Fatemi, H., Aminifard, M. and Samane, M. 2013. Effect of post harvest salicylic acid treatment on fungal decay and some post harvest quality factors of kiwi fruit. Archives of Phytopathology and Plant Protection, 46(11): 13381345.

Ferguson, I.B. 1984. Calcium in plant senescence and fruit ripening. Plant Cell Environment, 7: 477-489.

Hajilou, J. and Fakhimrezaei, S. 2013. Effect of post harvest calcium chloride or salicylic acid treatments on the shelf life and quality of apricot fruit. The Journal of Horticultural Science and Biotechnology, 88(5): 600-601.

Han, T.Y., Wang, L. Li. and X. Ge. 2003. Effect of exogenous salicylic acid on post harvest physiology of peaches. In: R.K. Prange, XXVI International Horticultural Congress: Issues and Advances in postharvest Horticulture, Canada, 12 December, 2003. Acta Horticulturae, 628pp.

Illeperuma, C.K. and Jayasuriya, P. 2002. Prolonged storage of 'karuthacolomban' mango by modified packaging at low temperature. Journal of Horticultural Science and Biotechnology, 77(2): 153157.

Kang, G.Z., Wang, G.C. and Sun, G.C. 2003. Participation of $\mathrm{H}_{2} \mathrm{O}_{2}$ in enhancement of cold chilling by salicylic acid in banana seedlings. Acta Botanica Sinica, 45: 567-573.

Leslie, C.A. and Romani, R.J. 1988. Inhibition of ethylene biosynthesis by salicylic acid. Plant physiology, 88: 833-837.

Lester, G.E. and Grusak, M.A. 2004. Post harvest application of calcium and magnesium to honeydrew and netter muskmelons: Effects on tissue ion concentrations, quality and senescence. Journal of American Society of Horticultural Science, 124: 545-552. 
Lougheed, E.S., Murr, D.P. and Miller, S.R. 1979. Effect of calcium and daminozide on ethylene production and softening of apple fruits. Experimentia, 35: 43-44.

Lu, X.H., Sun, D.Q., Mo, Y.W., Xi, J.G. and Sun, G.M. 2010. Effect of post harvest salicylic acid treatment on fruit quality antioxidant metabolism in pineapple during cold storage. The Journal of Horticultural Science and Biotechnology, 85(5): 454-458.

Manoj, K.S. and Upendra, N.D. 2000. Delayed ripening of banana fruit by salicylic acid. Plant Science, 158: 8796.

Marboh, E. 2009. Influence of postharvest treatments on shelf life and quality of litchi (Litchi chinensis Sonn.) cv. Rose Scented. Thesis, M.Sc.(Ag.) Horticulture, G. B. Pant University of Agriculture and Technology, Pantnagar. p.132.

Murthy, S.K. and Rao, K.P.G. 1982. Regulation of ripening by chemicals in "Alphonso" mango. Journal of Horticultural Science, 16(2): 179-183.

Ozeker, E. 2005. Salicylic acid and its effects on plants. E.U. Faculty of Agriculture Journal, 42(1): 213-223.

Poovaiah, B.W. 1984. Role of calcium in prolonging storage life of fruits and vegetables. Food Technology, 40: 8689.

Ray, P.K., Rani, R. and Singh, S. K. 2004.
Effect of temperature and sulphur treatments on storage behaviour of litchi fruits. Indian Journal of Horticulture, 61(4): 292-295.

Salunkhe, D.K. and Desai, B.B. 1984. Post harvest biotechnology of fruits. 1:43-57. Boca Raton: CRC Press.

Sayyari, M., Balabar, M., Kalantari, S., Serrano, M. and Valero, D. 2009. Effect of salicylic acid treatment on reducing chilling injury in stored pomegranates. Postharvest Biology and Technology, 55(3): 152-154.

Vendrell, M. 1970. Acceleration and delay of ripening in banana fruit tissue by gibberellic acid. Australian Journal of Biological Sciences, 23: 553-559.

White, P.J. and Broadley, M.R. 2003. Calcium in plants. Annals of Botany, 92: 487-511.

Wills, R.B.H., Bembridge, P.A. and Scott, K.J. 1980. Use of flesh firmness and other objective tests to determine consumer acceptability of delicious apples. Australian Journal of Experimental Agriculture and Animal husbandry, 20: 252-56.

Yalpani, N., Enyedi, A.J. and Raskin, I. 1994. Ultraviolet light and ozone stimulate accumulation of salicylic acid pathogenesis related proteins and virus resistance in tobacco. Planta, 19: 372376.

\section{How to cite this article:}

Afreen Tabasum, Ch. Raja Goud, Veena Joshi, D. Anitha Kumari and Bhagwan, A. 2019. Effect of Post Harvest Treatments on the Shelf life and Quality of Guava [Psidium guajava (L.)] cv. Allahabad Safeda. Int.J.Curr.Microbiol.App.Sci. 8(01): 2686-2697. doi: https://doi.org/10.20546/ijcmas.2019.801.283 